\title{
Dulwich OnView: an art museum-based virtual community generated by the local community
}

\author{
Ingrid Beazley \\ Dulwich Picture Gallery \\ Gallery Road \\ London SE21 7AD \\ United Kingdom \\ ingrid@beazley.netkonect.co.uk
}

\author{
Jonathan P. Bowen \\ Museophile Limited \\ Oak Barn, Sonning Eye \\ Reading RG4 6TN \\ United Kingdom \\ jpbowen@gmail.com
}

\author{
Alison H.Y. Liu \\ National Taiwan Normal University \\ No. 19, Da-Tong West Road \\ Taoyuan City \\ Taiwan, R.O.C. \\ sunny601101@gmail.com
}

\author{
Sarah McDaid \\ London South Bank Univ. \\ Faculty of Business \\ Borough Road \\ London SE1 OAA, UK \\ sarah.mcdaid@/sbu.ac.uk
}

\begin{abstract}
Dulwich OnView (DOV) is a museum blog magazine run by the Friends of Dulwich Picture Gallery (DPG) for the surrounding community in south London. It includes articles concerning both the gallery and the local community, helping to promote DPG in the process, in a fast-moving informal way that complements the official DPG website. For example, a competition for online artworks has been undertaken using DOV in association with a temporary exhibition of works by the $20^{\text {th }}$ century British artist Paul Nash. This paper provides an overview of the progress of the blog, since its launch in January 2008, including statistical information. A redesign of the DOV blog has been undertaken in 2010, aimed at a better structuring of the increasing number of articles into categories and the integration between the more formal and static DPG website and the more informal and dynamic DOV website, with suitable interconnecting links. As part of the redesign process for the DOV website a usability review was conducted. DOV has also been part of a wider study, investigating a number of varied museum-based virtual communities in an art education context using the social science Community of Practice (CoP) framework.
\end{abstract}

Virtual community. Art gallery. Social networking. Blogging. Web design.

\section{BACKGROUND}

As the web has expanded and interactive facilities have improved, social interaction online has increased rapidly over the last decade. Initially much of the interaction was professional in nature (Bowen et al., 2003). Individual cultural institutions including museums have tried to harness this technology for their own ends in engaging with their audiences with varying success (Beler et al., 2004; Russo \& Watkins, 2008). Curators themselves are also using social media (Decker, 2010). Such facilities have also been used for collaborative artistic endeavours (Smith, 2009), but sustaining such audiences has proved more difficult unless the right motivation to revisit can be found (Russo \& Peacock, 2009).

There are a number of different Web 2.0 technologies available for collaborative support. For example, wikis are a suitable technology for projects that involve collaboration and have been utilised in a museum context in interesting and varied ways, even if to a limited extent so far (Bowen et al., $2007 \&$ 2008). Blogs are more suitable for an ongoing magazine-like facility depending on a temporal ordering of articles and associated comments. For developing a virtual community, this is an appropriate technology to use as a basis for support facilities. Dulwich OnView is an example of such a blog-based magazine in the cultural and specifically arts sector (see Figure 1).

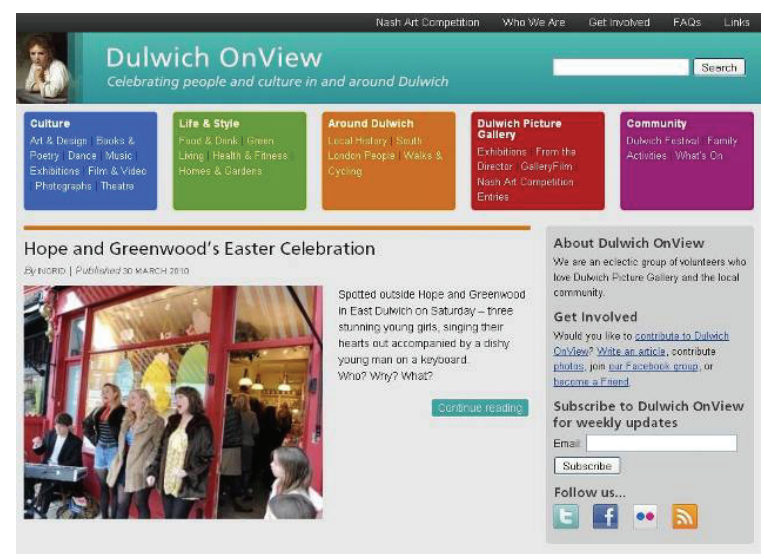

Figure 1: Dulwich OnView home page in 2010 (http://www.dulwichonview.org.uk). 


\section{DULWICH ONVIEW}

Dulwich OnView (DOV), founded in 2008 (Beazley, 2008a; Beazley, 2008b), has grown out of a very strong local community in south London (Holt, 2010). Dulwich, now a London suburb, still has the feeling of the village it once was, within the suburban expanse of south London. Located within this 'village' is the well-established Dulwich Picture Gallery (DPG), founded in 1811 in a purpose-built building. It holds a small but important collection of European old master paintings, mainly from the $17^{\text {th }}$ and $18^{\text {th }}$ centuries. It also hosts temporary exhibitions. For example, in 2010, a retrospective of the $20^{\text {th }}$ century British artist Paul Nash has been held (see Figure 2).

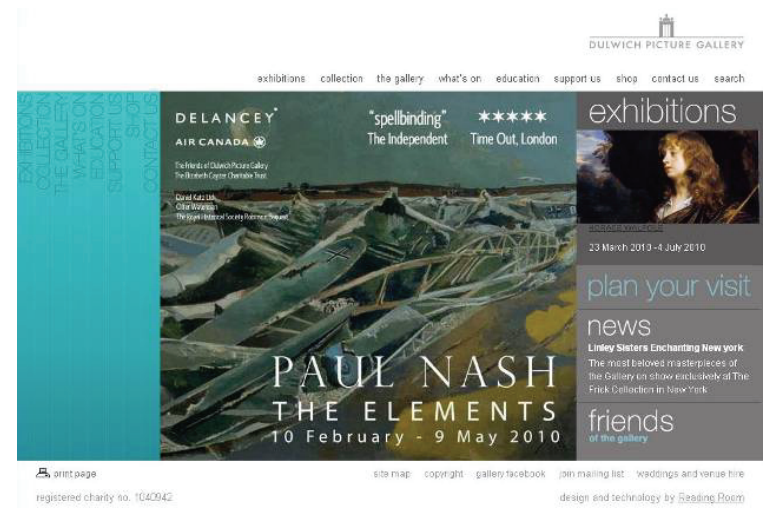

Figure 2: Dulwich Picture Gallery home page, including the Paul Nash exhibition in 2010

(http://www.dulwichpicturegallery.org.uk)

Dulwich OnView (DOV) is a unique example of a museum blog magazine run by volunteers from the Friends of Dulwich Picture Gallery (DPG) and the local community in Dulwich, with articles concerning both the museum and the local community. This combination attracts a far wider range of readers than a website exclusively about DPG. Visitors to DOV may not necessarily have heard of DPG, but are introduced to it via the numerous articles and links. (evidence from web traffic). This comes at effectively no cost to DPG. DOV is sometimes seen as an 'unofficial' museum website for DPG (Beazley, 2009) and this informality has allowed the site to be a thriving and dynamic facility for the local community in Dulwich, with a strong connection to DPG.

\subsection{Objective of DOV}

Articles relating to DPG are informal, sometimes quirky and personal, and always supportive. Since these articles are written by independent members of the community and not by the DPG marketing department, they are far more believable and effective in marketing terms. In addition, many members of the DPG staff, including the director, enjoy writing informally about the background to exhibitions, and their role in general. They are able to do this on DOV. Using their own personal, informal styles which are inappropriate for the main DPG website, they are recognised as individuals rather than in their executive roles.

DOV readers often communicate with Gallery staff via the comment boxes, something that does not happen on the DPG site. The director has had online conversations with researches as far afield as Australia, Canada and the US.

DOV fills these gaps in the service provision of DPG since DOV is able to act independently from DPG, but in a trusted role due to its links through the Friends of DPG.

\subsection{Implementation}

DOV has been implemented by a core group of volunteer editors with a varied skill set. The expertise needed, and that has been gathered, includes editorial, management, journalistic, photographic, filmmaking, networking, and technical skills. The magazine has been implemented using the standard WordPress blog publishing technology that is freely available online (http://www.wordpress.org). The aim is to minimise costs and maximise the free use of local people as volunteers. This has been very successfully achieved during over two years of operation.

The ever-growing number of articles on the DOV website is an increasingly important resource and the vast majority of them are still relevant. There has been a web interface redesign to allow easier access to the articles by dividing the magazine into sections. One of these is specifically dedicated to DPG to emphasise DOV's links to DPG

\subsection{Organisation}

DOV has many volunteers involved with its running to a greater or lesser extent. There is a core of editors and writers who administer and contribute to the magazine. Informal meetings are held to organise the management of DOV, and the possibility of real-world social networking as well as online community building is a significant incentive for the volunteers involved.

There are two interns working for DOV, one of whom continues to edit even though she has moved to Brussels. The other is a third year student in Arts Management at London South Bank University and her work with DOV is part of her coursework. A school student has written some articles before returning to school. Other contributors have included school leavers about to start university and young people wishing to work in illustration, who provide material for articles. DOV 
acts as a platform for three authors and two professional photographers, giving them online exposure and the prestige of being associated with DPG, even if indirectly. Recently there has been an 80 year old regular contributor and conversations have built up between her and 2 other octogenarians via the comment boxes.

Articles are submitted for publication via email on an entirely voluntary basis with no payment involved. There are a number of regular writers, but anyone may submit an article. These are checked by an editor and then scheduled for publication on a Tuesday or Friday each week. Articles are normally only lightly copy-edited if needed and a chatty informal and personal style is encouraged.

\subsection{Statistical evaluation}

The original DOV website went live in January 2008. Since then, DOV has seen steadily increasing visitor numbers (see Figure 3 ). It can be noted that December and January are slightly quieter months each year. There is anecdotal evidence that when there are blog posts about future events, ticket sales suddenly increase.

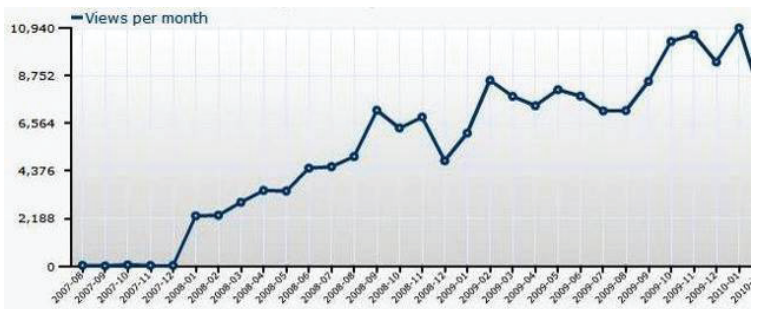

Figure 3: Dulwich OnView views per month

$28 \%$ of visitors arrive at DOV through reciprocal links from organisations about who DOV has written articles. 25\% come from local information websites and discussion forums. 14\% arrive from the main DPG website via many different links to DOV articles. $12 \%$ are from other blogs and $9 \%$ from Facebook, 4\% from Flickr, and $2 \%$ from the associated Twitter micro-blog (http://www.twitter.com/dulwichonview) - see Figure 4. In August 2009, a dedicated DOV entry on Wikipedia

(http://en.wikipedia.org/wiki/Dulwich OnView) was created, again by a volunteer, and $3 \%$ of visitors have come from this source, even though it is a relatively recent addition.

There are many outgoing links on DOV. 33\% of outgoing visitors follow a link to the DPG main website or Flickr site. $20 \%$ go to the DPG home page, the 'what's on' page or an exhibition page; the other $13 \%$ go to Flickr. The figure of $33 \%$ travelling from DOV to DPG compares to $14 \%$ in the opposite direction.

\section{About Dulwich OnView}

We are an eclectic group of volunteers who love Dulwich Picture Gallery and the local community.

\section{Get Involved}

Would you like to contribute to Dulwich

OnView? Write an article, contribute

photos, join our Facebook group, or

become a Friend.

Subscribe to Dulwich OnView for weekly updates

Email

\section{Subscribe}

Follow us...

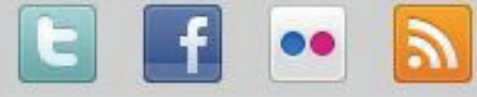

Figure 4: About Dulwich OnView, including links to Twitter, Facebook, and Flickr

With regard to search terms, 'Dulwich OnView' and variants are not the most popular keywords in practice. In fact, 'flamenco' is top with $22 \%$. There are articles on flamenco and it is likely that this page has been linked from another popular website or published in some effective way. Variants of 'Dulwich On View' are only the third most popular search term at $11 \%$. Explicit searches for DOV have increased with time. The term 'Dulwich Picture Gallery' is substantially down the list of popular keywords at $2 \%$. Even though $98 \%$ of visitors arriving at the DOV website through a search have not selected 'Dulwich Picture Gallery', $33 \%$ of onward clicks go to DPG, which is a free benefit for the Gallery.

The site is the subject for a study in the context of an ongoing postdoctoral study of online museumrelated community websites in the context of the social science Community of Practice framework, along with four other internationally renowned museums and art galleries. The redesign of the web interface has been subjected to a formal usability review study, through voluntary but professional effort. DOV will be demonstrated again at the Museums and the Web conference in Denver, USA, in April 2010, and more detailed information on the two studies will be available in an associated paper on the MW2010 website (http://www.archimuse.com/mw2010). 


\subsection{Unique features}

DOV is different from other museum-based blogs in a number of ways. Importantly, it is independent from the museum. DOV is run by the community for the community, with DPG at its heart. This gives it a sense of belonging within the local area and makes it part of people's everyday lives. The DOV website is discovered and used by people not looking for (and perhaps not knowing about) DPG. However, they become introduced to DPG through the associated articles and numerous links on the website. DOV is not dominated or controlled by official authority, although it still benefits from formal recognition and support from DPG. This makes the content relating to DPG, created by independent volunteers, more believable, giving it the ability to gain public trust and further involvement from the local community. DOV uses no DPG resources and DPG staff is under no pressure to submit articles on a regular basis, although they do so when time permits. DOV encourages anyone to contribute content. Although a limited number of people have editorial access, hundreds have written articles and produced photographs and even films for this hybrid blog/magazine. Contributors come from a far wider demographic than the physical visitors to DPG. These contributors often tell their peers about their article, who then in turn discover DPG, using the viral effect that networked social media enables.

\subsection{Cost-effectiveness}

Dulwich OnView is run with minimal budget by volunteers, in association with the Friends of Dulwich Picture Gallery. There are no financial costs to DPG itself. The costs for the web technology are minimal, and are covered by sponsorship from local businesses or directly by the editors. All contributors are volunteers, so this promotional tool comes at no cost burden at all to DPG. Hence it is an extremely cost-effective way of publicising DPG, with an effectively infinite return on investment for the Gallery in financial terms.

\section{ONLINE ART COMPETITION}

DOV, in conjunction with a special exhibition on the British artist Paul Nash at DPG in 2010, organised an art competition for artworks inspired Dulwich Picture Gallery in Nash style. Realistically artists could not create entries without visiting DPG, so one of the competition's purposes is to increase the numbers of real visitors. The competition is featured on a dedicated page directly accessible as prominent tab link at the top of the DOV home page (see Figure 5).

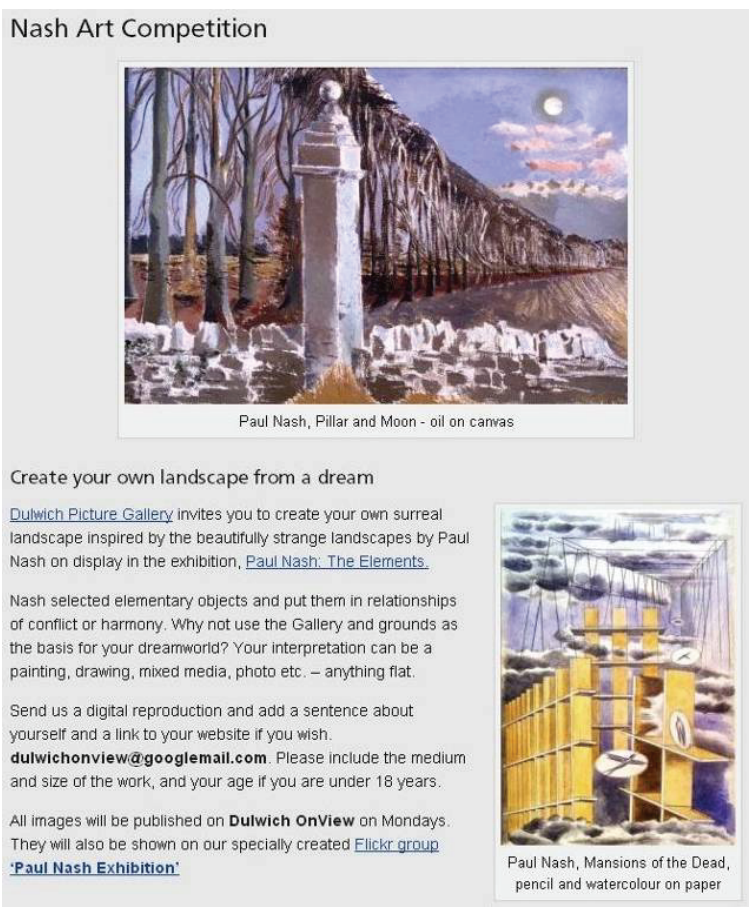

Figure 5: Dulwich OnView Nash Art Competition (www.dulwichonview.org.uk/dpg-online-art-competition)

Submissions of individual artworks have been featured as blog entries, like other articles, on the DOV website (e.g. see Figure 6).

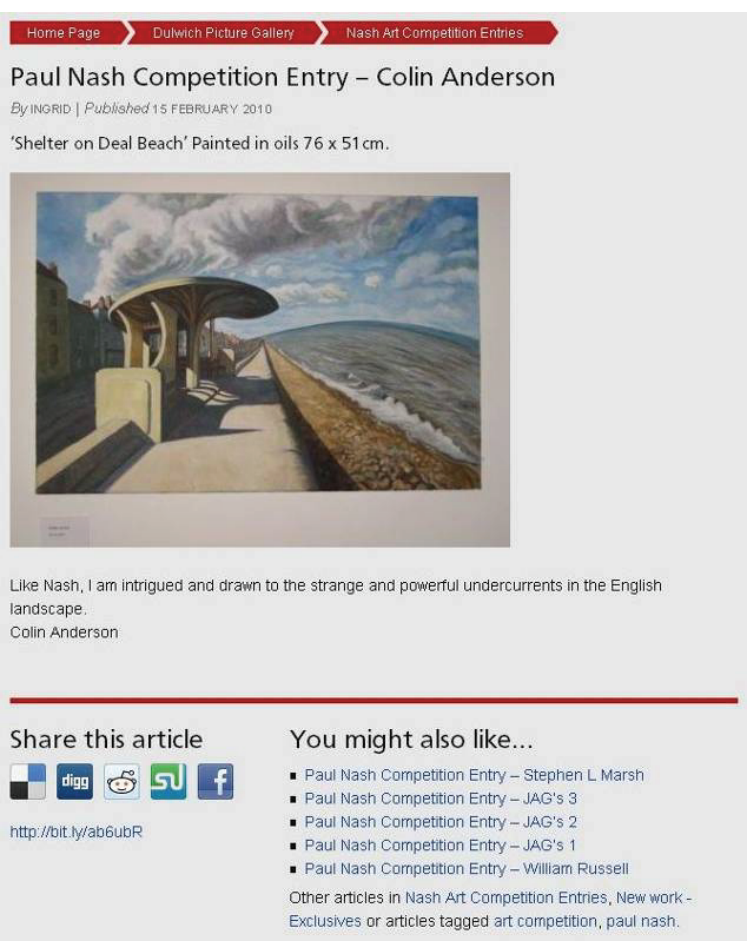

Figure 6: Paul Nash competition entry (Colin Anderson)

As with many blogs, comments are possible for these competition entries. For example, see Figure 
7 for two positive comments and the possibility of leaving a further comment concerning the entry in Figure 6. Comments are moderated by DOV editors for safety and appropriateness, There is a $24 / 7$ editorial rota, so comments are always moderated within hours. Entries are also available on the Flickr website (see Figure 8).



Figure 7: Paul Nash competition comments for Colin Anderson's competition entry

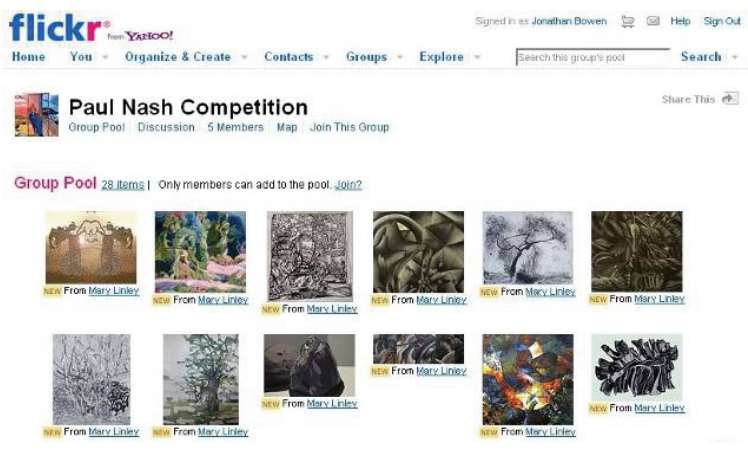

Figure 8: Paul Nash Competition entries on Flickr (http://www.flickr.com/groups/1313937@N24)

The winners are chosen by the numbers of votes in the comment boxes associated with the individual entries, thus encouraging further community involvement with DPG and the exhibition.

In this section, an example of the use of DOV for an online art competition has been presented. In the next section, the activities of DOV are considered more formally in the framework of a Community of Practice. This concept is increasingly being applied to virtual communities as well as real communities (Varlamis \& Apostolakis, 2010).

\section{A COMMUNITY OF PRACTICE}

Dulwich OnView has been selected as one of a set of wider case studies for a PhD thesis (Liu, 2008 \& 2009). The study investigates a number of varied museum-based virtual communities using the social science Community of Practice (Wenger et al., 2002) as its framework. This in-depth case study includes several that are run by national museums (Liu \& Bowen, 2010). DPG is relatively small-scale, but the study concludes that DOV has greatly benefited its associated museum as many of its activities conform to the principles of a Community of Practice (CoP).

There are three fundamental elements forming the structural model of a CoP, namely domain, community, and practice. Wenger et al. (2002, p. 29) state that: 'When they function well together, these three elements make a community of practice an ideal knowledge structure - a social structure that can assume responsibility for developing and sharing knowledge'. DOV as a virtual community to a large degree embraces these three elements that give it the potential of being cultivated as a successful community of practice.

In terms of domain, a CoP must have a common interest to be effective. Otherwise, it is just a collection of people with no particular purpose participating in the group. At first glance, the domain knowledge in the DOV community seems not very definite. There is a far broader variety of articles that go beyond the Gallery-related topics being included the discussions within the community, which makes a clearly defined domain less obvious. Nevertheless, DOV indicates in the Who we are web page: 'From East to West Dulwich and beyond we wanted to recognise the eclectic nature of life round here, the breadth of people and livelihoods, the rude health of the Arts, and the buoyant sense of community...'. This online community does create a common ground that attracts local people coming to celebrate the shared culture within their physical neighbourhood.

With regard to community, a CoP needs a group of people who are willing to engage with some others in the group, so ultimately the entire group is transitively connected as a unified entity. This aspect is critical to the effective development of shared knowledge within the community. As a hybrid blog/online magazine, DOV encourages everyone to contribute content to the community, including comments on articles, which has enabled many local residents to feel proud of being authors, and hence build a sense of ownership in it.

In terms of practice, a CoP explores existing common knowledge whilst also developing practice 
for the future. The term 'practice' denotes a set of socially defined approaches of doing things in the shared domain that create a baseline for communication and action within the group. As the domain knowledge of DOV more or less engenders local people's emotional attachment in the Dulwich area and their motivation for participating in the community, fostering a strong sense of community that can hold continuing interactions is indeed achievable.

Developing a healthy CoP requires the interplay of these three elements within a community in a balanced manner, because they are all dynamically changing over time. Whilst it is important to have the three elements controlled to a degree in a CoP, perseverance in one element will help ease the potential problems in another. As Wenger et al. (2002, p. 47) point out: 'If the domain is clear and the practice is well established, then people can come and go without harming the community'. Albeit that this did happen to the DOV community at times, the whole community still has continued to evolve healthily and prosperously as time has progressed.

The success or failure of a community of practice largely depends on the objectives of the community combined with its interests and resources. Wenger et al. (2002, chap.3) have identified seven specific aspects that should be addressed to enable a CoP to flourish. Nevertheless, these principles are recurrent aspects of the life of a CoP itself, rather than external rules that are to be imposed on the community. More detailed examples of how DOV conforms to these principles can be found in Liu et al. (2010).

\section{WEBSITE REDESIGN}

The Dulwich OnView website interface was redesigned in 2010 to provide better organisation of the articles available on the site. As part of the redesign process, a usability review was conducted (McDaid, 2009). A usability review is just one tool available to a usability expert for use during an iterative user-centred design process. The aim of the review is to identify those aspects of a design that might affect the overall usability of a website. Usability can be defined as 'the extent to which a product can be used by specified users to achieve specified goals with effectiveness, efficiency and satisfaction in a specified context of use' (ISO, 1998). Usable websites support a user in achieving their goals without them for example, becoming frustrated, anxious or having negative feelings towards the site.

The report (McDaid, 2009) made several recommendations regarding the overall layout of the designs and highlighted both those elements that met usability guidelines and those that might usefully be revisited. As the review was conducted on paper-based design wireframes, the interaction in terms of response times, etc., could not be fully evaluated. However, attention was drawn to factors such as the use of large or numerous images which might impact on the page download times.

Many features of the design were found to be broadly inline with usability guidelines. In particular, the organisation's name, logo and tagline were clearly visible and appropriately located on the homepage. The tagline was succinct and enabled visitors to quickly identify the purpose of the site. Other usable elements included the grouping together of similar items, the provision of a 'search' function and links to recent, popular, and archive posts incorporating fully spelled out month names to avoid confusion over date formats for non-UK visitors.

Importantly, the page designs also contained elements that would increase the website's trustworthiness. These included information about the site's creators, how to contact them and short biographies of the post authors. The report also suggested that retaining a 'privacy policy' similar to the one on the current site would further increase visitor trust.

However, the review did highlight two important usability issues. These related to the allocation of screen space for the different sections of the webpage (main content, navigation, banners, etc.) and inconsistencies in the location, organisation and labelling of the main navigation links.

The new DOV website was implemented in March 2010 and the revised design (see Figures 1 and 9) addresses many of the findings contained in the usability report. Specifically, the concern regarding the irregular placement and naming of navigational areas has been rectified and a light background colour with high-contrast text has been used to facilitate online reading.

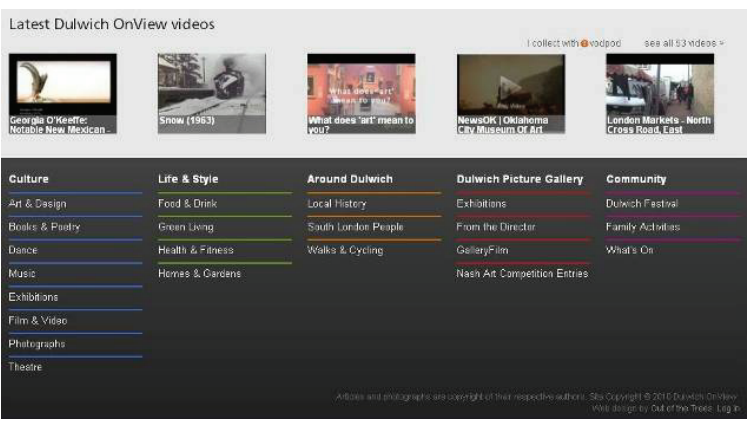

Figure 9: New DOV footer, including video links 
Most DOV submissions are in the form of short text articles with pictures, but there are also videos on the DOV website, linked from the footer section of pages (e.g., see Figures 9 and 10).

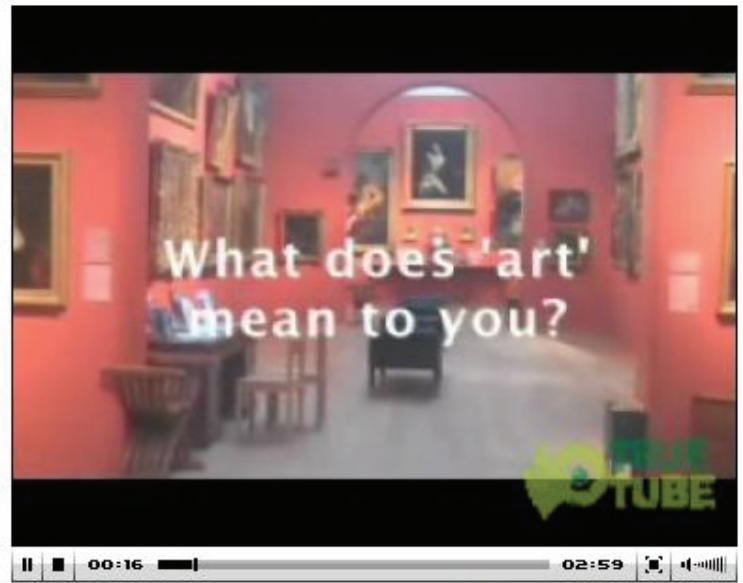

Figure 10: Video on DOV website

Based on comments to the blog to date, the new website has been well received. Taking into account that regular users tend not to welcome any change to a familiar website, this suggests that the DOV team has successfully focused on the needs of its online community during the redesign process. Further information on the usability review and the previous interface can be found in Liu et al. (2010).

\section{CONCLUSION}

This paper has presented the online blog/magazine Dulwich OnView (DOV), associated with Dulwich Picture Gallery (DPG). It provides a flourishing online community with a capability to present mainly articles, but also videos, in an informal and friendly setting, with a cultural and artistic flavour.

In 2010, a competition to create artworks in the style of Paul Nash has been undertaken on DOV in association with an exhibition at DPG. Public voting has been enabled using the comment boxes at the end of each article, with a prize at the end of the exhibition. This is being promoted via DPG's emailing list amongst other places.

The paper has also overviewed DOV in the context of a Community of Practice (CoP), which includes three fundamental elements, seven major principles, and five stages of community development. All of these apply to DOV to a large degree so although CoP is mostly seen in a scientific, engineering, business, or educational context, the model can be extended to the museum field as well.
A redesign of the website in 2010 has helped in the organisation of the content on DOV, as the number of articles has increased and needs better organisation and categorisation. A usability review of a proposed web interface has been undertaken and the findings have been used to help improve the newly redesigned website.

The DOV facility is believed to be unique amongst museums and galleries with its thriving online community promoting an art gallery within a local neighbourhood and beyond. It is a model that other museums could follow if there is an existing real community and at least one 'champion' that has the time and ability to take a sustained lead role and who has the complete trust of the management.

In summary, the informality and activity of DOV contrasts well and appropriately with the formality and slower pace of DPG's website. In summary, DOV is a distinctive example of a thriving local community website based around an existing wellestablished art gallery. Both benefit greatly from the existence of each other, with little burden on the gallery. It is hoped that this successful example will encourage others to attempt similar online artsbased social communities in the future.

\section{ACKNOWLEDGMENTS}

This paper is a development of a paper associated with a demonstration at the Museums and the Web 2010 conference (Liu et al., 2010). Thank you to Ian Dejardin, the Director of Dulwich Picture Gallery, for helpful discussions with the authors in December 2009. Jonathan Bowen is also a Visiting Professor at the University of Westminster and an Emeritus Professor at London South Bank University (LSBU). Alison H.Y. Liu was a Visiting Research Fellow at LSBU during 2009, which aided her study of DOV and DPG. In addition, thank you to Professor Jo Chiung-Hua Chen in National Taiwan Normal University for her continuous support and encouragement.

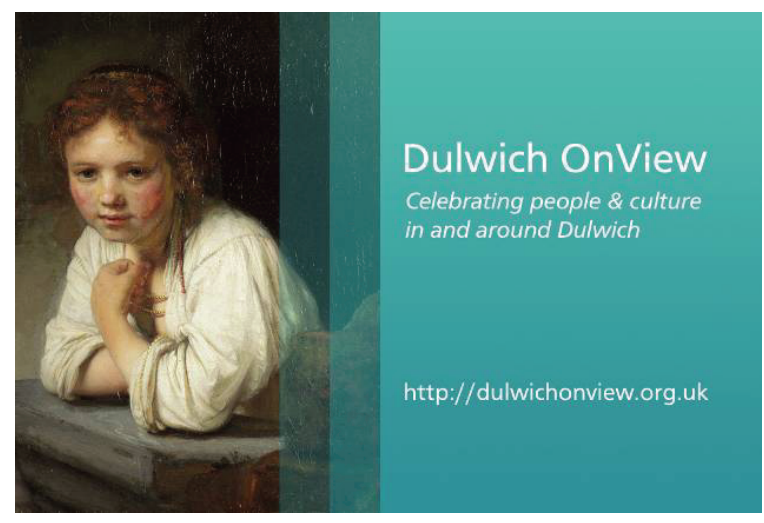

Figure 11: Dulwich OnView business card 


\section{REFERENCES}

Beazley, I. (2008a) Reach new audiences, increase numbers of visitors, and become a major part of the local community by using online social networking sites and blogs. In Museums and the Web 2008: Demonstrations. Archives \& Museum Informatics, Toronto.

Beazley, I. (2008b) What are you waiting for? BAFM Journal, Issue 92, page 19, Summer. British Association of Friends of Museums.

Beazley, I. (2009) The unofficial museum website. BAFM Journal, Issue 95, p. 17, Summer. British Association of Friends of Museums.

Beler, A., Borda, A., Bowen, J.P., and FilippiniFantoni, S. (2004) The building of online communities: An approach for learning organizations, with a particular focus on the museum sector. In J. Hemsley, V. Cappellini, and G. Stanke (Eds.), EVA 2004 London Conference Proceedings, University College London, The Institute of Archaeology, UK, 26-30 July, pp. 2.12.15 .

Bowen, J.P., Houghton, M., and Bernier, R. (2003) Online museum discussion forums: What do we have? What do we need? In D. Bearman and J. Trant (Eds.). Museums and the Web 2003: Proceedings. Toronto: Archives \& Museum Informatics.

Bowen, J.P., Bernal, I., Filippini-Fantoni, S., and Lisney, E. (2007) A Museums Wiki. In J. Trant and D. Bearman (eds.), Museums and the Web 2007: Proceedings. Archives \& Museum Informatics, Toronto.

Bowen, J.P. (2008) Wiki software and facilities for museums. In J. Trant and D. Bearman (Eds.), Museums and the Web 2008: Proceedings. Archives \& Museum Informatics. Toronto.

Dicker, E. (2010) The impact of blogs and other social media on the life of a curator. In D. Bearman and J. Trant (eds.). Museums and the Web Conference 2010, Denver, USA, 13-17 April. Archives \& Museum Informatics, Toronto.

Holt, J. (2010) Case Study: Dulwich Picture Gallery. Museum Practice, 49, p. 52, Spring.

ISO (1998) BS EN ISO 9241-11:1998 Ergonomic requirements for office work with visual display terminals (VDTs) - Part 11: Guidance on usability. International Organization for Standardization.
Liu, A.H.Y. (2008) Using online communities to attract museum visitors. International Journal of Education through Art 4(3), pp. 259-274, December. DOI: 10.1386/eta.4.3.259_1

Liu, A.H.Y. (2009) Creating online learning environments for museums. Museology Quarterly 23(3), pp. 5-35.

Liu, A.H.Y. and Bowen, J.P. (2010) Creating online collaborative environments for museums: A case study of a museum wiki. International Journal of Web Based Communities. Accepted for a special issue on Designing Shared Spaces for Collaborative Creativity.

Liu, A.H.Y., McDaid, S., Bowen, J.P., and Beazley, I. (2010) Dulwich OnView: A museum blog run by the community for the community. In D. Bearman and J. Trant (Eds.). Museums and the Web Conference 2010, Denver, USA, 13-17 April. Archives \& Museum Informatics, Toronto.

McDaid, S. (2009) Dulwich OnView Usability Report, Usability Design Report. London, UK, October.

Russo, A. and Watkins, J. (2008) New literacy new audiences: Social media and cultural institutions. In S. Dunn, S. Keene, G. Mallen, and J.P. Bowen (Eds.), EVA London 2008 Conference Proceedings, 22-24 July, pp. 225-239. Electronic Workshops in Computing, British Computer Society.

Russo, A. and Peacock, J. (2009) Great expectations: Sustaining participation in social media spaces. In J. Trant and D. Bearman (Eds.), Museums and the Web 2009: Proceedings. Archives \& Museum Informatics, Toronto.

Smith, S. (2009) The creative uses of Facebook as a tool for artistic collaboration. In A. Seal, S. Keene, and J.P. Bowen (Eds.), EVA London 2009 Conference Proceedings, 6-8 July, pp. 181-191. Electronic Workshops in Computing, British Computer Society.

Varlamis, I. and Apostolakis, I. (2010) Selfsupportive virtual communities. International Journal of Web Based Communities, 6(1), pp. 4361. DOI: 10.1504/IJWBC.2010.030016

Wenger, E., McDermott, R.A., and Snyder, W. (2002) Cultivating Communities of Practice: $A$ guide to managing knowledge. Harvard Business School Press, Boston. 